\title{
Medical Journal of Shree Birendra Hospital: Journey Continues.
}

\author{
Amit Joshi ${ }^{1}$ \\ ${ }^{1}$ Executive Editor MJSBH \\ Department of Orthopedics, Shree Birendra Hospital.
}

Journal is considered as an indicator of research activities and prestige of an institution, hence there has been a trend to publish a medical Journal by almost all teaching institution. Very rarely, a non-teaching hospital was found to publish a Medical Journal. Medical Journal of Shree Birendra Hospital (MJSBH) is unique not only because it is published by a hospital but also one of the oldest medical journal of our country ${ }^{1}$. Since its first issue in 1999 (2056 BS), hospital has tried to make this journal a scientific document and has achieved certain milestones also, but still far from gaining a reputation of a good scientific medical journal. For any journal to achieve a good reputation, various parameters are required, important of them are: consistency and regularity in publication, good quality manuscript, excellent visibility, constant flow of articles, good editorial team and a sound financial backup ${ }^{2}$. This editorial has tried to analyze the above mentioned parameters of MJSBH, so that future planning could be done to improve the quality of MJSBH.

CONSISTENCY AND REGULARITY OF PUBLICATION: During its initial days MJSBH used to be published once a year with very few research articles. Majority of articles were personal experiences or summary of a chapter. Although most of the articles could not be given any evidence level but there was an effort to publish it regularly and the publication was regular till 2003. There was no issue on 2004, but a major breakthrough was made in 2005, when a souvenir MJSBH took a shape of medical Journal. The 2005 and 2006 issues came up with many improvements not only in quality of articles but also the editorial process. For the first time in the history of MJSBH, author's guideline has been given and the scope of the journal was made clear. But after that there was no publication for next three years. Again from 2010, the Journal was resurrected with more definitive goal of achieving scientific recognition. Since 2010 the journal became biannual, and trying to achieve consistency and become regular in future. Timely publication has been a real problem throughout the years, now the journal is trying to have a dedicated staff so that, editorial process could be monitored closely and processed swiftly. Year 2012 was again a milestone for MJSBH, as it was decided to publish as an official publication of Nepalese Army Institute of Health Sciences (NAIHS). This association with NAIHS will help the journal in may aspect including the regularity in publication.

MANUSCRIPT QUALITY: A good quality of article is always a dream of any journal, but not all the journals are fortunate. MJSBH has tried to incorporate all kind of articles in the past just to encourage the authors for writing. This may be the reason for having evidence level for most of our articles below IV (Table 1). If we carefully analyze our articles, it is evident that almost all of our articles have no level of evidence in initial years, but since 2010 the journal has stopped publishing articles which could not be categorized to any level of evidence (Table 1). A brighter aspect of our journal is improvement in type of articles. During its initial issues, most of its articles were unclassified, but gradually the number of unclassified articles decreased and MJSBH started publishing only original articles, review articles and case reports (Table 2). From this issue onwards we strictly accepted original articles and cases reports with review of literature only, and hope to improve the quality of published article in future also.

Table 1. Evidence level of articles published in MJSBH.

\begin{tabular}{|c|c|c|c|c|c|c|}
\hline Year & I & II & III & IV & V & No LE \\
\hline 1999 & 0 & 0 & 0 & 0 & 3 & 13 \\
\hline 2000 & 0 & 0 & 0 & 0 & 1 & 13 \\
\hline 2001 & 0 & 0 & 0 & 0 & 1 & 9 \\
\hline 2002 & 0 & 0 & 0 & 0 & 4 & 15 \\
\hline 2003 & 0 & 0 & 0 & 0 & 5 & 16 \\
\hline
\end{tabular}




\begin{tabular}{|c|c|c|c|c|c|c|}
\hline 2004 & \multicolumn{6}{|c|}{ No publication } \\
\hline 2005 & 0 & 0 & 0 & 1 & 8 & 8 \\
\hline 2006 & 0 & 0 & 0 & 1 & 11 & 5 \\
\hline 2007 & \multirow{3}{*}{\multicolumn{6}{|c|}{ No Publication }} \\
\hline 2008 & & & & & & \\
\hline 2009 & & & & & & \\
\hline $2010-1$ & 0 & 0 & 0 & 1 & 7 & 1 \\
\hline $2010-2$ & 0 & 0 & 0 & 1 & 9 & 0 \\
\hline 2011 - 1 & 0 & 0 & 0 & 2 & 8 & 0 \\
\hline 2011 - 2 & 0 & 0 & 0 & 6 & 4 & 0 \\
\hline
\end{tabular}

Table2. type of articles

\begin{tabular}{|c|c|c|c|c|c|}
\hline Year & Original & Case report & Review & Unclassified & Remarks \\
\hline 1999 & 1 & 2 & 0 & 13 & 16 \\
\hline 2000 & 1 & 0 & 0 & 13 & 14 \\
\hline 2001 & 1 & 0 & 0 & 9 & 10 \\
\hline 2002 & 2 & 3 & 0 & 14 & 19 \\
\hline 2003 & 2 & 3 & 0 & 16 & 21 \\
\hline 2004 & \multicolumn{5}{|c|}{ No publication } \\
\hline 2005 & 6 & 2 & 1 & 8 & 17 \\
\hline 2006 & 2 & 8 & 2 & 5 & 17 \\
\hline 2007 & \multirow{3}{*}{\multicolumn{5}{|c|}{ No Publication }} \\
\hline 2008 & & & & & \\
\hline 2009 & & & & & \\
\hline $2010-1$ & 3 & 5 & 0 & 1 & 9 \\
\hline $2010-2$ & 7 & 3 & 0 & 0 & 10 \\
\hline $2011-1$ & 7 & 3 & 0 & 0 & 10 \\
\hline $2011-2$ & 5 & 4 & 1 & 0 & 10 \\
\hline
\end{tabular}

VISIBILITY OF JOURNAL: Visibility is one of the most important factors to give any credibility to the journal. Although MJSBH is not having its own dedicated website, but since 2010, MJSBH is made online with the help of NepJol(http:// nepjol.info/index.php/MJSBH). Recently the links of MJSBH has been incorporated in web page of Nepalese Army Institute of Health Sciences (http://www.naihs.edu.np/) and Shree Birendra Hospital (http://www.birendrahospital.mil. $\mathrm{np} /$ index.php) also. This has definitely increased the visibility of our journal.

CONSTANT FLOW OF ARTICLES: In the past, MJSBH was some what unfortunate in constant flow of articles. There have been time when there is a long queue of article, and sometimes the editorial board has to repeatedly request for articles. From this issue onwards MJSBH has opened door for articles even from outside our institution. On the other hand with the addition of Basic Science faculties through NAIHS, will help in having continuous flow of articles in future. Since the establishment of NAIHS, hospital will need many more faculty members in different specialties. According to Trivuwan University rule the faculty members must have certain publications under their name to be promoted from one position to another, this will also have an impact on continuous flow of article.

GOOD EDITORIAL TEAM: MJSBH is very fortunate to have dedicated editorial team from very beginning. During its initial days, the editor had to work really hard to compile the articles and it has to be approved by the higher authorities in military hierarchy. Daunting individual effort was required in earlier years. Now, the editorial board is all inclusive and have mixture of experience and enthusiasm. During past few years trained editors have regularly featured in editorial board and their experience are instrumental in shaping the MJSBH to a true medical journal. From 2010 onwards, the editorial board has prepared a pool of peer reviewers who are very helpful in improving the quality of article. Peer reviewing has become an integral part of editorial process of all the articles submitted to MJSBH.

FINANCIAL SUPPORT: Initial issues of MJSBH were financially supported by the advertisement on the journal, provided by various companies. From this issue onwards, the MJSBH is also an official publication of NAIHS. The administrative 
authorities are trying to sort out the problems of finance and provide a constant financial support for MJSBH. Till date there are no permanent office for the journal. Recommendations have been made to provide an editorial office, which will improve the service to authors and facilitate the review process.

Future of MJSBH: Journey so far has not been smooth for MJSBH. Despite a 13 years glorious history, the distance travelled by this medical journal is very minimal. Expectations were high from very beginning, it continuously raised and it has reached to a level where tremendous effort has become mandatory. Irony of situation is that the institutional support alone is just not enough. MJSBH requires high quality articles which have to come from authors. MJSBH has already laid down guidelines for authors and even prepared templates to facilitate authors for correct submission of articles, but even until this issue, only $30 \%$ of the articles were according to the format of MJSBH. The editorial board had to spend lot of time to bring the manuscript in to the format. This clearly indicates that there is a need of training to authors. MJSBH has already conducted a one day seminar on "how to write a research paper?" and planning to conduct many more in future in association with Nepalese Association of Medical Editors (NAME). however, only attending seminars and training again will not be enough if there are no interest on doing research and publishing it. So, a research culture and writing attitude has to be developed within ourselves and this is not a responsibility of institution. Institution should facilitate and create an environment to conduct the research and MJSBH is always there to help our authors to publish and make their work visible by wider group of people.

\section{REFERENCES:}

1. Archives of Medical Journal of Shree Birendra Hospital.

2. Jain AK, Kumar S. Indian Journal of Orthopedics: Journey Countious. Indian J Orthop. 2013;47:1-5. 